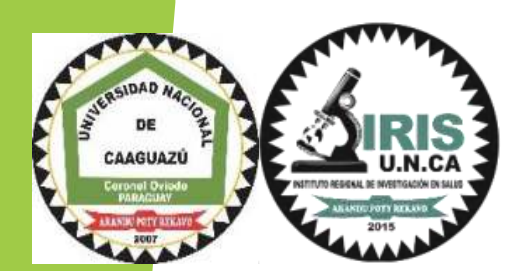

\title{
Risk behaviors of syphilis among indigenous population in Paraguay. 2017
}

*Estigarribia G. ${ }^{\text {, }, ~ A g u i l a r ~ G . ~}{ }^{1,2}$; Miranda AE ${ }^{2}$, Mc Farland W³, Ortiz A. ${ }^{1}$, Kawabata A4., Rios $\mathrm{P}^{1}, \mathrm{Muñoz} \mathrm{S}^{5}$ Samudio T

Regional health research institute, National University of Caaguazu ${ }^{1}$, Federal University of Spirito Santo $^{2}$, Global Health Sciences, University of California, San Francisco, California, USA ${ }^{3}$, National HIV Program, Paraguay ${ }^{4}$, Department of Public Health, University of the Frontier, Temuco, $\mathrm{Chile}^{5}$

\section{Background}

Paraguay has reported a syphilis prevalence of $4 \%$ however, information is scarce on the epidemiology of these infections among indigenous people by and regarding which linguistic families should be targeted by specific prevention efforts. The objective of the current study was to determine the risk behaviors across the populations of indigenous in ParaguayMethods: We conducted a cross-sectional survey among indigenous of the 5 linguistic families in Paraguay in 2017. Probable, multistage random sampling. Participants complete a questionnaire on demographic characteristics and behavior, and blood samples were drawn for rapid testing for syphilis and TPPA confirmation. Participants provided written informed consent. A multivariable analysis was used to identify factors associated with syphilis infection (socio-demographic, condom use, multiple couples, alcohol use at last sexual intercourse , transactional sex).

\section{Results}

A total of 3018 indigenous were enrolled. The largest proportion of trans women enrolled (66.1\%) were women, $74.2 \%$ had educational assistance.The majority (57.3\%) was aged between 20 and 44 years old. The syphilis prevalence was $6.93 \%$ (95\% confidence interval 16.04 7.89). In the multivariable analysis, indigenous with 25 years and older ( $\mathrm{aOR}=2.18 ; 95 \% \mathrm{Cl} 1.14 ; 4.16)$, multiple couples (aOR=1.80; 95\% IC 1.25;2.61), alcohol use at last sexual intercourse $(\mathrm{aOR}=1.28 ; 95 \%$ IC $0.88 ; 1.89$ and transactional sex (aOR=3.22; 95\% IC 1.62;6.41).were significantly more likely to be infected with syphilis.

\section{Conclusion}

Our survey found high prevalence of syphilis in indigenous population. There is an urgent need to implement strategies that reduce the risk behaviors that include an intercultural adaptation, designed according to the context and language of the indigenous population in Paraguay.

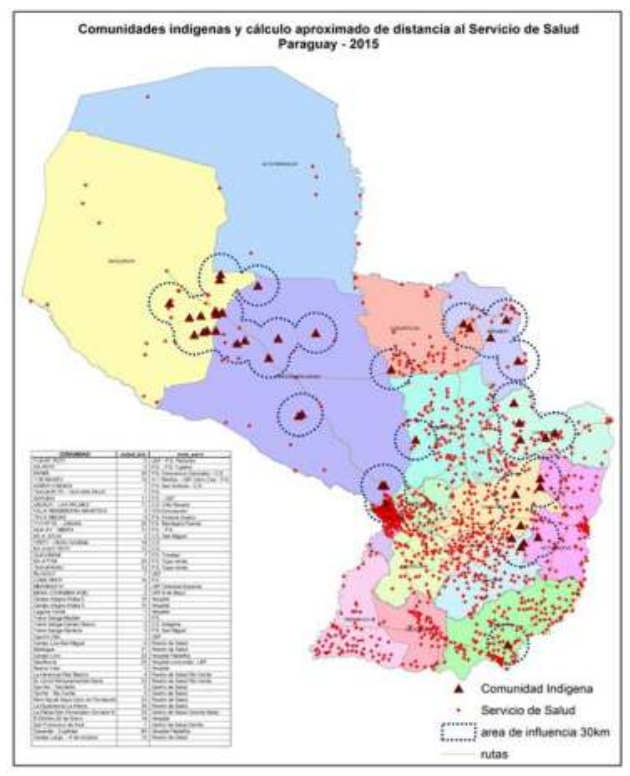

Prevalence of syphilis, by linguistic families, 2017

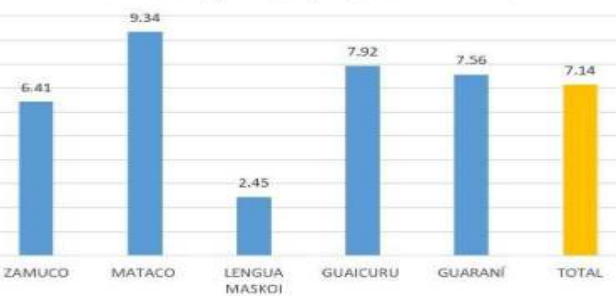

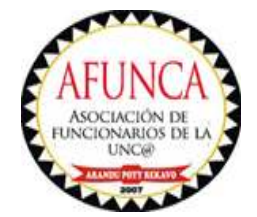
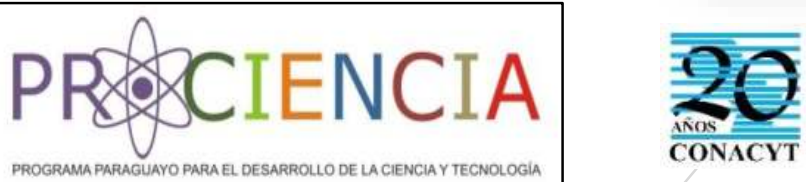